\title{
Radical coupling allows a fast and tuned synthesis of densely packed polyrotaxanes involving $\gamma$-cyclodextrins and polydimethylsiloxane
}

\author{
F. Blin ${ }^{1}$, C. Przybylski ${ }^{1 \dagger}$, V. Bonnet ${ }^{2}$, M. J. Clément ${ }^{3}$, P.A. Curmi ${ }^{3}$, P. Choppinet $^{4}$, T. \\ Nakajima $^{4}$, H. Chéradame ${ }^{1}$, N. Jarroux ${ }^{1 *}$ \\ ${ }^{1}$ Laboratoire Analyse et Modélisation pour la Biologie et l'Environnement, Université d'Evry-Val d'Essonne, \\ CNRS UMR 8587, Bâtiment Maupertuis, bd F. Mitterrand, 91025 Evry, France. \\ ${ }^{\dagger}$ Present address: Institut Parisien de Chimie Moléculaire, Université Pierre et Marie Curie, CNRS UMR 8232, 4 \\ place Jussieu, F-75252 Paris Cedex 05, France. \\ ${ }^{2}$ Laboratoire de Glycochimie, des Antimicrobiens et des Agroressources, Université de Picardie Jules Verne, \\ CNRS UMR 7378, 33 rue Saint-Leu, 80000 Amiens, France. \\ ${ }^{3}$ Laboratoire Structure-Activité des Biomolécules Normales et Pathologiques, Université Evry-Val d’Essonne, \\ INSERM UMR 829 Evry 91025, France. \\ ${ }^{5}$ Menicon Co., Ltd; Applied Technology Development Department, Kasugai 4870032, Japan. \\ Email:nathalie.jarroux@univ-evry.fr \\ Phone number: +33 (0) 1-69-47-77-19. Email: nathalie.jarroux@univ-evry.fr.
}

\section{Supporting Information}

\section{Table of content :}

Figure S1. SEC chromatograms of polyrotaxane from run 1 to 5.

Figure S2. Positive MALDI-TOF mass spectrum in linear (A) and reflector (B) mode obtained with sinapinic acid (SA) of the purified product by preparative SEC of run 5 .

Figure S3. 1D ${ }^{1} \mathrm{H}$ NMR spectra of (A) pseudo-polyrotaxane and (B) pure polyrotaxane $\quad$ p.4 extracted from run 5 .

Figure S4. 1D ${ }^{13} \mathrm{C}$ NMR spectra of polyrotaxane obtained from run $4(\mathrm{~A})$ and $5(\mathrm{~B}) . \quad$ p.5

Figure S5. 2D HSQC spectra of polyrotaxane obtained from run 4 (A) and 5 (B). p.6 


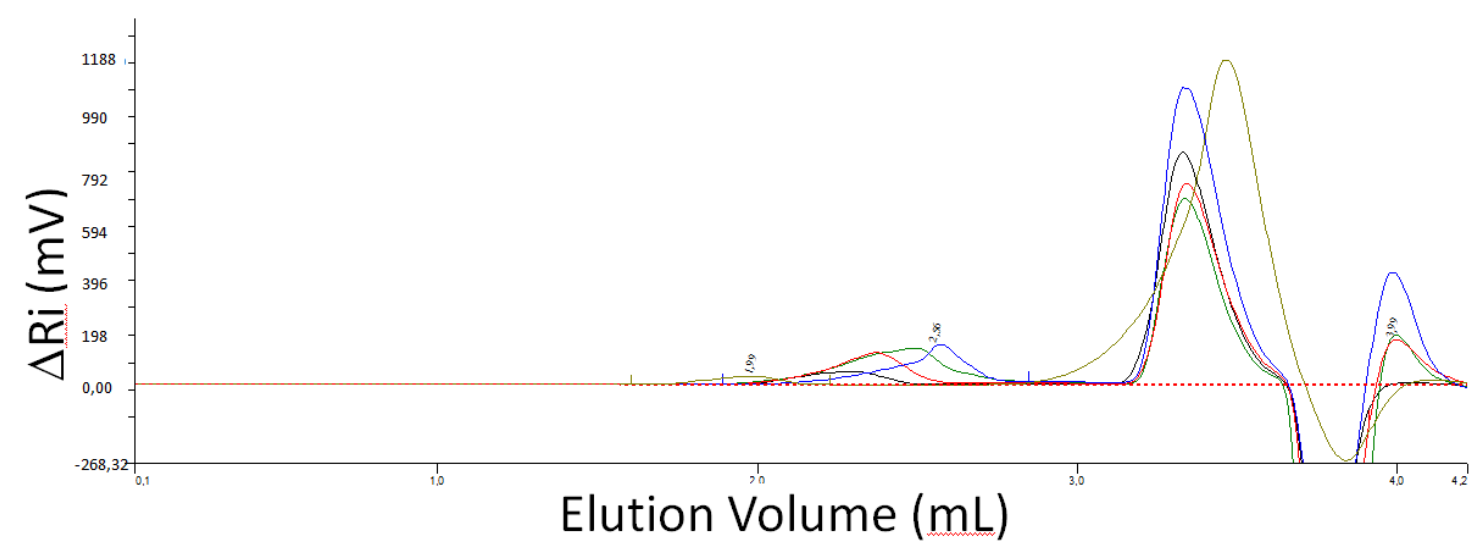

Figure S1. SEC chromatograms of polyrotaxane from run 1 to 5 using analytical column analyzed in DMF as run 1 (light green trace), run 2 (black trace), run 3 (red trace), run 4 (blue trace) and run 5 (green trace). 
A

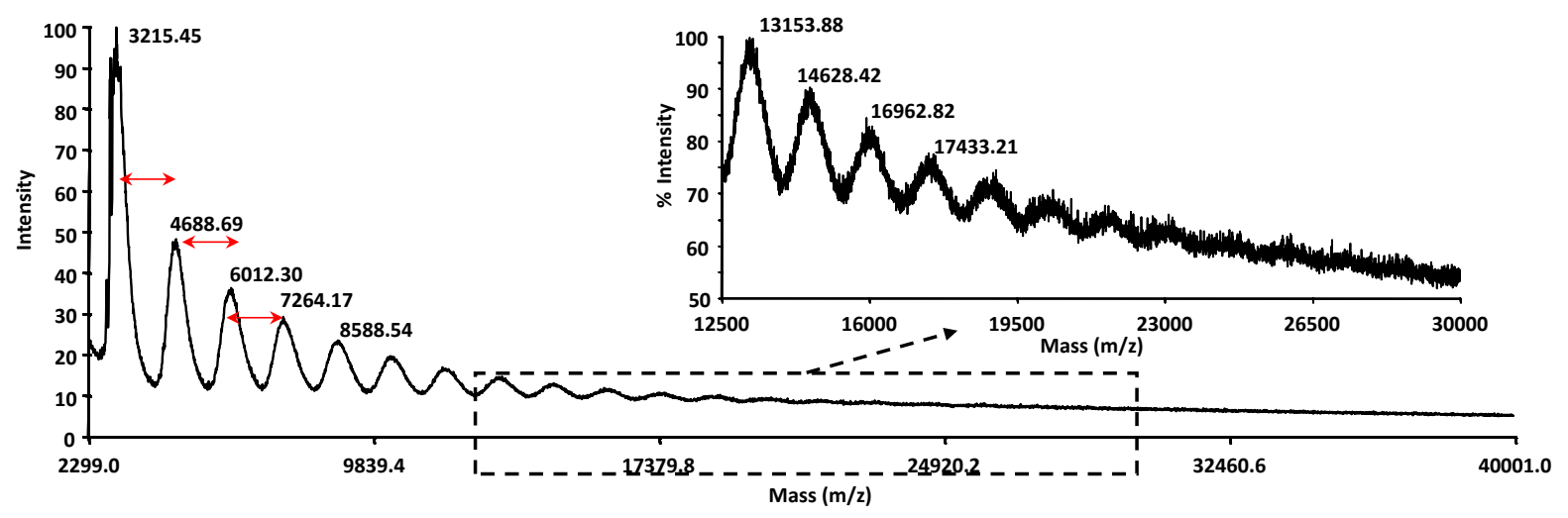

B

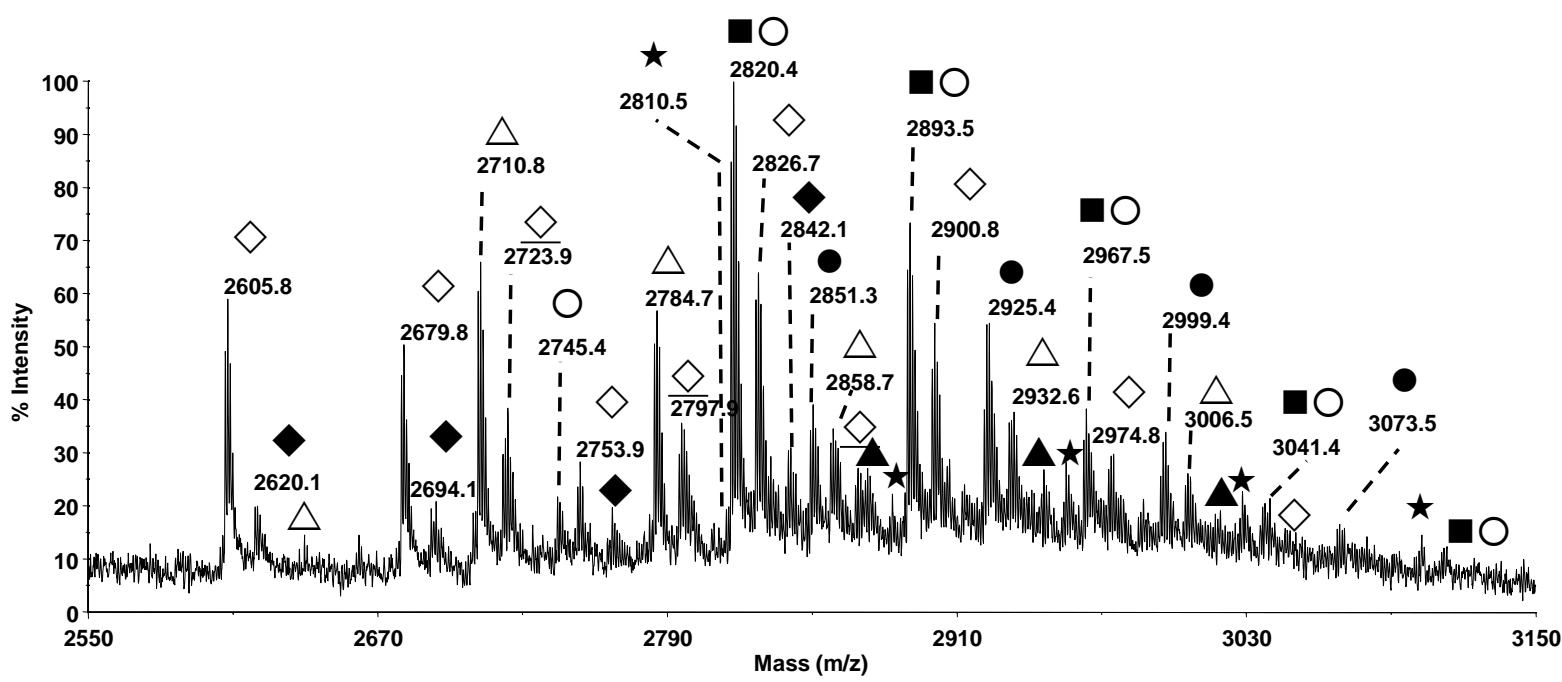

Figure S2. Positive MALDI-TOF mass spectrum in linear (A) and reflector (B) mode obtained with sinapinic acid (SA) at $20 \mathrm{mg} / \mathrm{mL}$ in methanol/water $1 / 1(\mathrm{v} / \mathrm{v})$ of the purified product by preparative SEC of run 5 . In linear mode, the inset shows the high mass range. In reflector mode, the identified series are : $\mathrm{Py}(\mathrm{H})-\left(\mathrm{OSi}\left(\mathrm{CH}_{3}\right)_{2}\right)_{\mathrm{n}}-(\mathrm{H}) \mathrm{Py}+1 \gamma \mathrm{CD}+\mathrm{H}^{+}(\mathbf{\Delta})$, $\mathrm{Py}(\mathrm{H})-\left(\mathrm{OSi}\left(\mathrm{CH}_{3}\right)_{2}\right)_{\mathrm{n}}-(\mathrm{H}) \mathrm{Py}+1 \gamma-\mathrm{CD}+\mathrm{Li}^{+}(\boldsymbol{\square}), \mathrm{Py}(\mathrm{S})-\left(\mathrm{OSi}\left(\mathrm{CH}_{3}\right)_{2}\right)_{\mathrm{n}}-(\mathrm{S}) \mathrm{Py}+1 \gamma-\mathrm{CD}+\mathrm{K}^{+}(\bullet)$, $\left(\mathrm{Py}(\mathrm{S})-\left(\mathrm{OSi}\left(\mathrm{CH}_{3}\right)_{2}\right)_{\mathrm{n}}-(\mathrm{S}) \mathrm{Py}+1 \gamma \mathrm{CD}+\mathrm{H}^{+}(\Delta), \mathrm{Py}(\mathrm{S})-\left(\mathrm{OSi}\left(\mathrm{CH}_{3}\right)_{2}\right)\right)_{\mathrm{n}}-(\mathrm{S}) \mathrm{Py}+1 \gamma-\mathrm{CD}+\mathrm{Na}^{+}$ $\left.(\underline{\oslash}), \mathrm{Py}(\mathrm{S})-\left(\mathrm{OSi}\left(\mathrm{CH}_{3}\right)_{2}\right)\right)_{\mathrm{n}}-(\mathrm{S}) \mathrm{Py}+1 \gamma-\mathrm{CD}+\mathrm{K}^{+}(\mathrm{O}), \mathrm{Py}(\mathrm{Py})-\left[\left(\mathrm{OSi}\left(\mathrm{CH}_{3}\right)_{2}\right]_{\mathrm{n}}-(\mathrm{Py}) \mathrm{Py}+1 \gamma-\mathrm{CD}\right.$ $+\mathrm{Li}^{+}(\star), \mathrm{Py}(\mathrm{Py})-\left[\left(\mathrm{OSi}\left(\mathrm{CH}_{3}\right)_{2}\right]_{\mathrm{n}}-(\mathrm{Py}) \mathrm{Py}+1 \gamma-\mathrm{CD}+\mathrm{Na}^{+}(\diamond)\right.$ and $\mathrm{Py}(\mathrm{Py})-\left[\left(\mathrm{OSi}\left(\mathrm{CH}_{3}\right)_{2}\right]_{\mathrm{n}^{-}}\right.$ $(\mathrm{Py}) \mathrm{Py}+1 \gamma-\mathrm{CD}+\mathrm{K}^{+}(\diamond)$. 
A

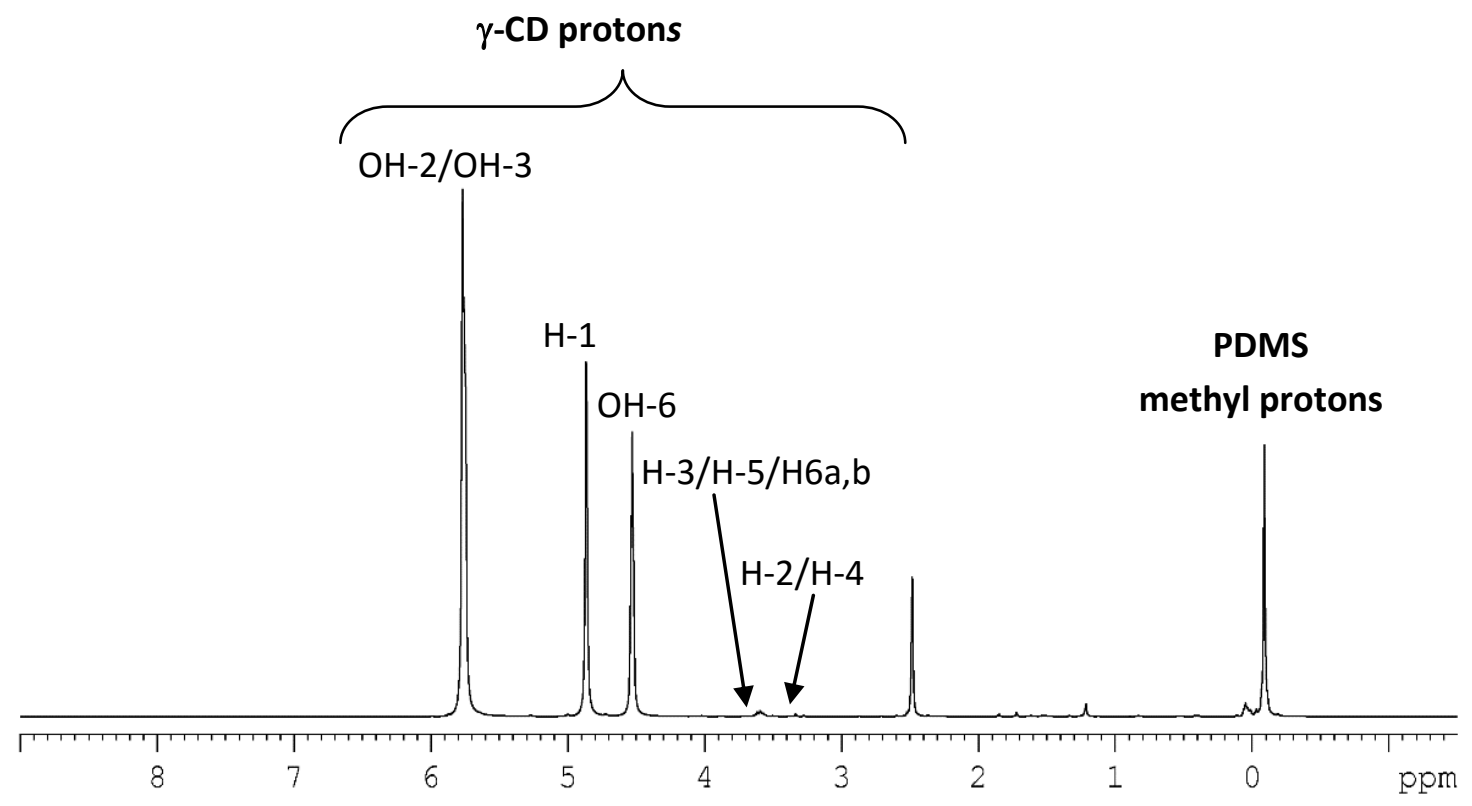

B

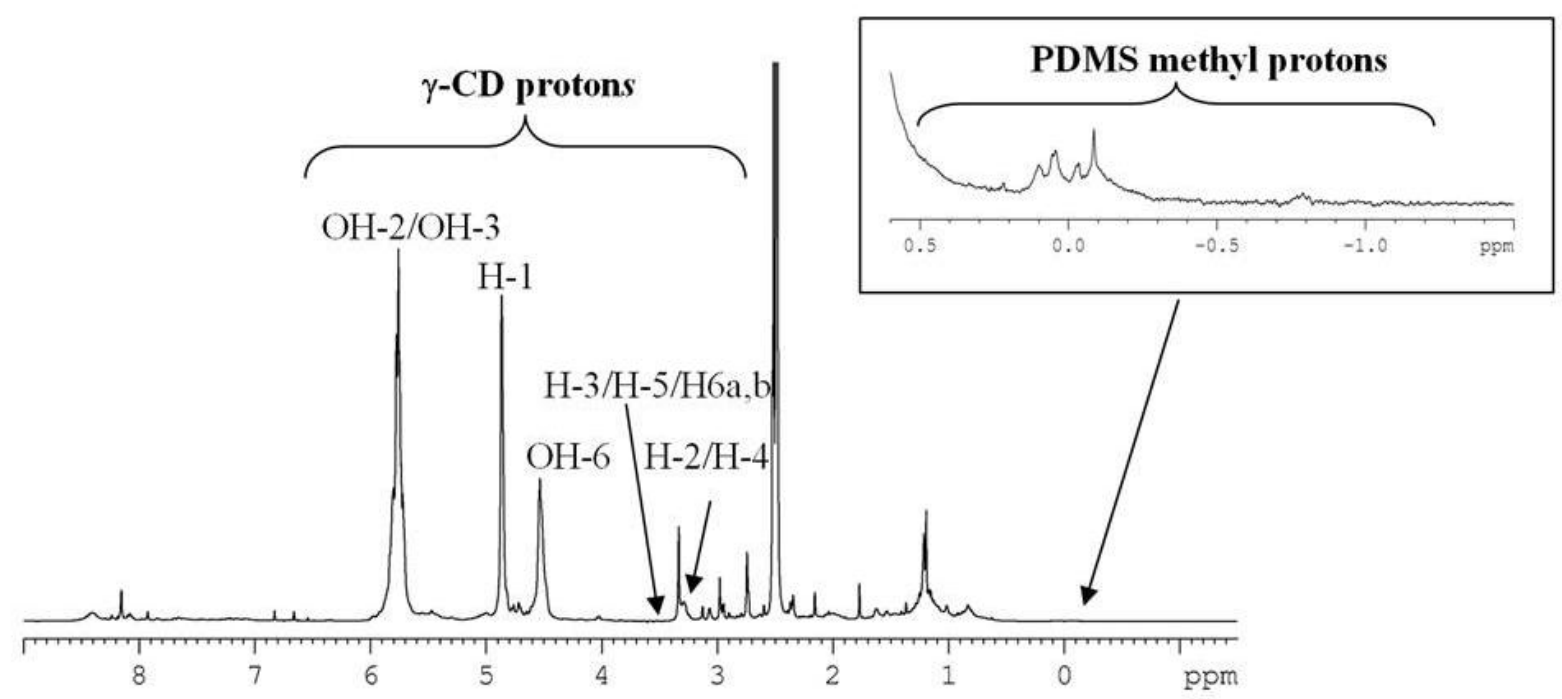

Figure S3. 1D ${ }^{1} \mathrm{H}$ NMR spectra $(600 \mathrm{MHz}$ with water signal suppression) of pseudopolyrotaxane (A) and PDMS alkyl protons area of pure highly threaded polyrotaxane (B) extracted from run 5, recorded at 298K in DMSO-d6. 
A

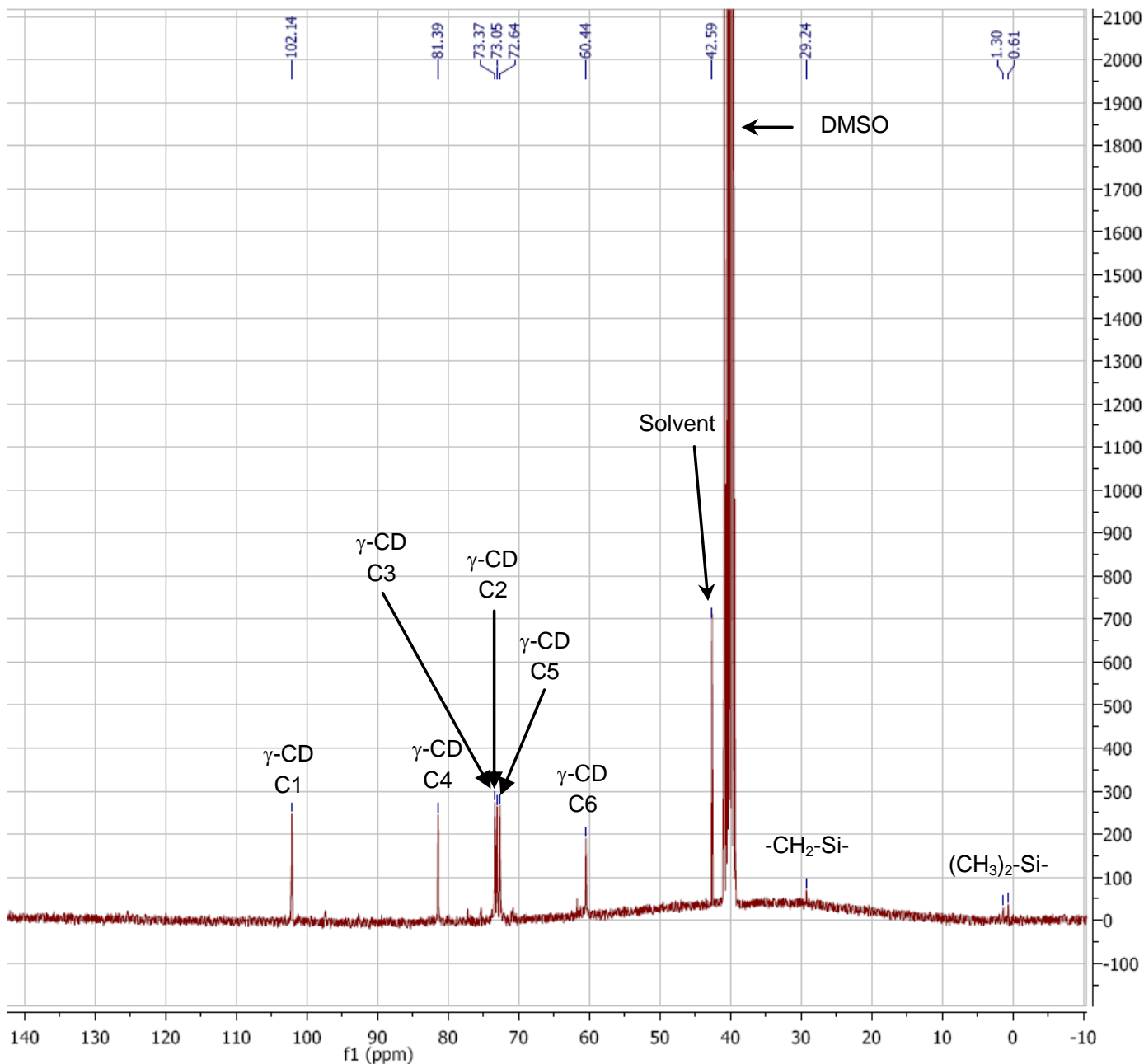

B

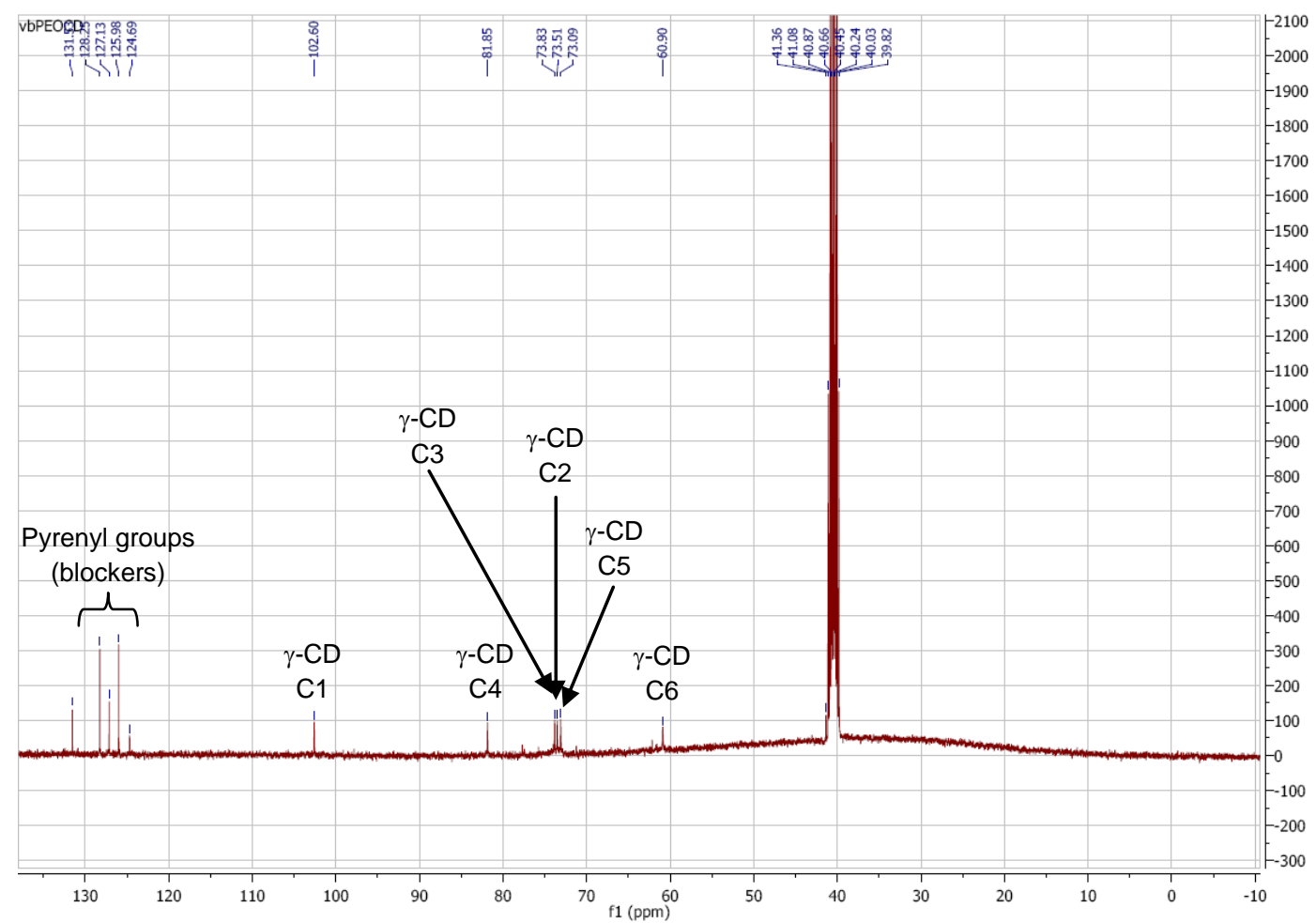

Figure S4. 1D ${ }^{13} \mathrm{C}$ NMR spectra of polyrotaxane obtained from run 4 (A) and 5 (B). Spectra recorded at $298 \mathrm{~K}$ in DMSO-d6 (100 MHz). 
A

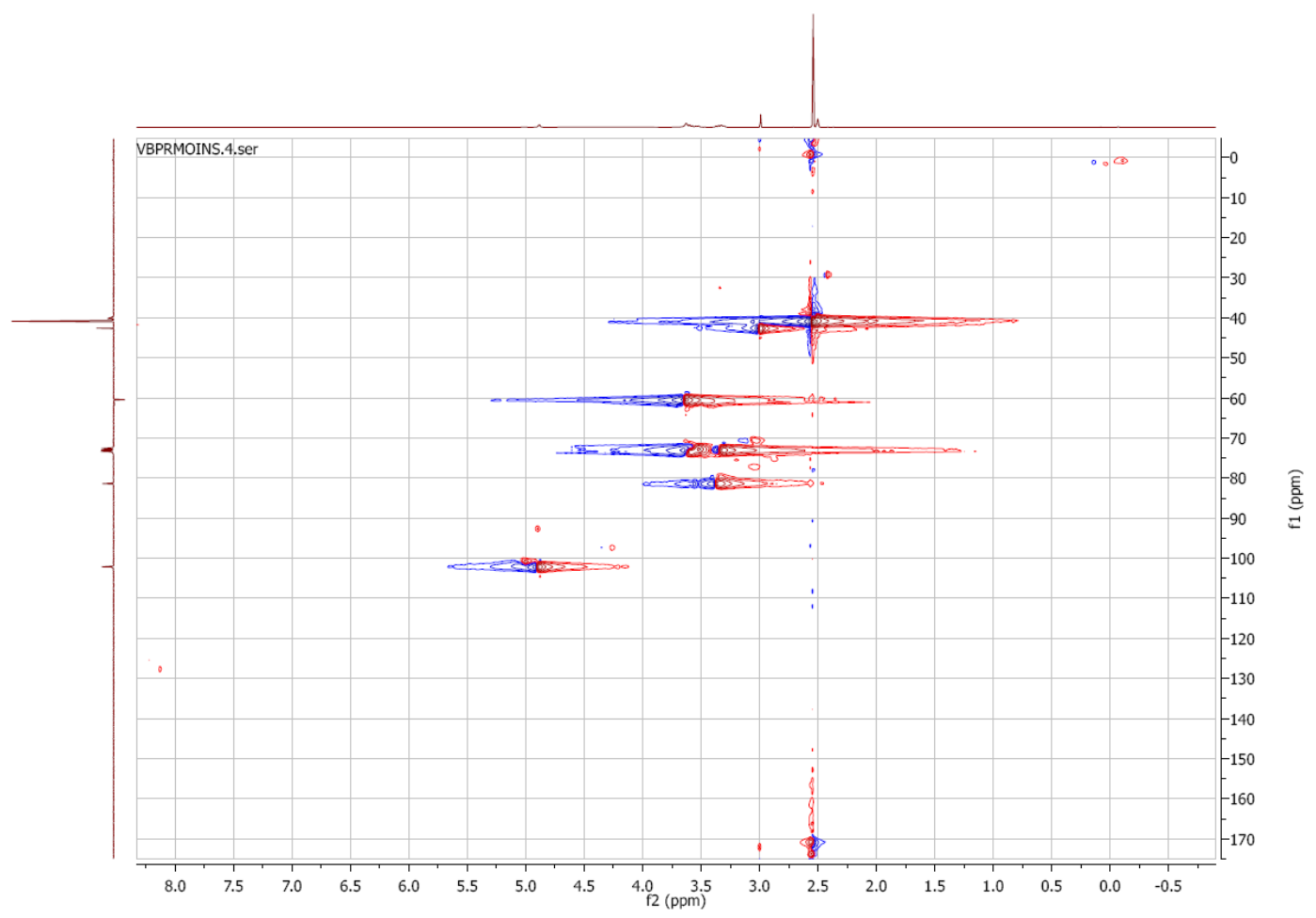

B

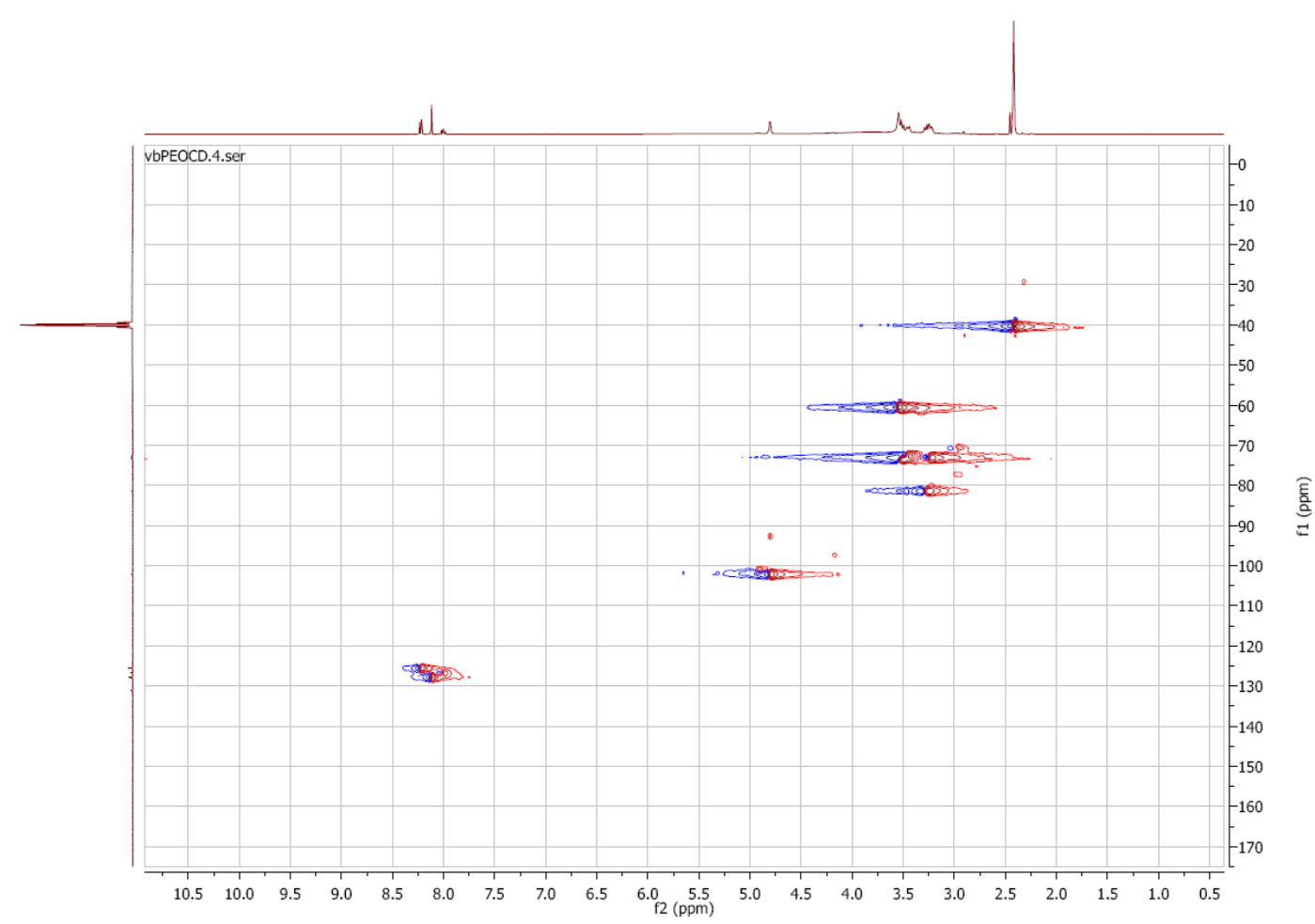

Figure S5. 2D HSQC spectra of polyrotaxane obtained from run 4 (A) and 5 (B). Spectra recorded at $298 \mathrm{~K}$ in DMSO-d6. 